\title{
The use of rebampid in celiac therapy as a possible reason for rapid complete remission. Clinical follow-
} up

\begin{abstract}
The purpose of the article: Demonstration of the case of rapid onset of clinical and histological remission in a patient with latent form of coeliac disease, with a syndrome of impaired suction of 3 degrees of severity with strict adherence to the agluten diet in combination with the intake of enteroprotector rebampid.

Material and methods: A 29-year-old woman was examined. Immunological tests were determined, the morphological structure of the small intestine mucosa was evaluated according to Marsh classification, the activity of intestinal enzymes (carbohydrase) by the method of A. Dalqvist.

Results: In the examination in the intestinal ward of the GBUS, the ICC identified high values of antibodies to tissue translutaminase, atrophy of the small intestine mucosa in the Marsh IIIC stage, the reduction of carbohydraz in the lining of the small intestine. Diagnosed coeliac disease, latent form, Disorder of disturbed suction 3 degrees of severity. Dysacharidase insufficiency. Appointed aglyuten diet (AGD), course reception of prednisone, was corrected metabolic disorders. After discharge: complied with the AGD. She finished the course of taking prednisone, for 2 months took rebampid. In the control examination after 2 months - clinical remission, restoration of the normal structure of SOTC, the level of carbohydraz.
\end{abstract}

Conclusion: The possibility of rapid remission under the influence of the cytoprotector Rebampid is discussed.

Keywords: aglyuten diet, metabolic disorders, enteroprotector rebampid, small body mucosa
Volume I 2 Issue 6 - 202I

\author{
Krums LM,Ahmadullin OV, Bykova SV, \\ Babanova AV, Parfenov Al \\ Loginov Moscow Clinical Research Center, Russia
} Correspondence: Larisa M Krums, Senior Researcher, Loginov
Moscow Clinical Research Center, Moscow, Russia, Email krumsml@gmail.com

Received: October 28, 2020 | Published: November 18, 2021

\section{Introduction}

Coeliac disease (gluten enteropathy) is an immuno-dependent disease characterized by the development of inflammation in the small body mucosa (SOTC) caused by the intake of cereals in hereditary predisposed persons. The mechanism of the damaging action of gluten is caused by both the toxic effect of it on SOTC people predisposed to coeliac disease, and the pathological reaction of T-lymphocytes to gluten. Today, the prevalence of coeliac disease in the adult population of most countries of the world is 1:100-1:250 of the total population. Among women, coeliac disease is 3-4 times more common than in men.

The disease always begins in childhood, but it can often be subclinical for a long time and manifest itself for the first time in adults or even in old age, this form is called the latent form of celiac disease. The factors provoking the appearance of the first clinical symptoms in women are most often pregnancy and childbirth. However, stressful situations, intestinal infections can act as provoking factors. ${ }^{3}$ One of the constant clinical symptoms is systematic diarrhea, accompanied by the development of a syndrome of impaired absorption, as well as increased gas production and symptoms of impaired absorption. ${ }^{4}$ An important role in the pathogenesis of impaired absorption is played by impaired digestion, assessed by a decrease in the activity of carbohydrases in the thin mucosa intestines. In the work of Akhmadullina O.V. it was shown that in patients with newly diagnosed celiac disease in $87 \%$ of cases, there is a decrease in the activity of carbohydrases: glucoamylase, maltase, sucrase, lactase. An inverse correlation was found between the degree of SOTC atrophy and the level of decreased activity of carbohydrases: with a decrease in the degree of SOTC atrophy, the level of intestinal enzymes in the mucous membrane increased. Changes in the activity of carbohydrases are of diagnostic and prognostic significance. ${ }^{5}$

The main treatment for celiac patients is strict, lifelong adherence to a gluten-free diet (AGD); with grade 3 malabsorption syndrome, patients are prescribed glucocorticoid therapy - intravenous prednisolone, followed by a switch to oral administration and a gradual withdrawal of the drug; correction of metabolic disorders is carried out. ${ }^{3}$

In numerous studies in recent years, the gastroprotective effect of the cytoprotector rebamipide, which promotes the healing of ulcerative lesions of the stomach and intestines, has been demonstrated. ${ }^{6-9}$ The mechanism of the protective action of rebamipide, its effect on reparative processes is explained by the stimulation of prostaglandin synthesis, the expression of growth factors, and improved epithelial proliferation..$^{10}$ There is evidence of a positive effect of rebamipide in bowel disease. As an example of the effectiveness of rebamipide, let us give the case history of patient $\mathrm{K}$. with latent celiac disease.

Patient K., aged 29, was admitted to the intestinal department of the A.S. Loginova in March 2018, with complaints of frequent heavy stools up to 10-15 times a day, bloating, rumbling in the abdomen, episodes of aching pain in the epigastric region after eating, swelling 
of the lower extremities, more in the hips, an increase in the volume of the abdomen, dry skin, hair loss, cramps and numbness of the limbs, weight loss by $15 \mathrm{~kg}$ over the past 3 months. He considers himself ill since June 2017, when, a month after giving birth, frequent, liquid, abundant stools appeared without pathological impurities. Hospitalized in the infectious diseases department of the regional (Kaliningrad) hospital with a diagnosis of acute gastroenteritis. The infectious agent has not been installed. She took enzyme preparations, astringents, the treatment had no effect. After discharge, the complaints persisted. There was a decrease in body weight, swelling of the legs. She was repeatedly treated in the gastroenterology department with a diagnosis of irritable bowel syndrome with diarrhea. Blood tests showed: anemia (hemoglobin - 100g/1), a decrease in the level of total serum protein $(36 \mathrm{~g} / 1)$. Treatment with 5 -aminosalicylic acid preparations, intestinal antiseptics, hepatoprotectors, and enzyme preparations was carried out. Against the background of transfusion of albumin, plasma, there was some improvement in the condition in the form of a decrease in edema. Diarrhea persisted and weight loss increased. Sent for examination and treatment at the A.S. Loginova. Hospitalized in the intestinal department of the A.S. Loginova DZM. On admission: a state of moderate severity. The physique is asthenic. Height $170 \mathrm{~cm}$. Weight $54 \mathrm{~kg}$. The body mass index is $18.69 \mathrm{~kg} / \mathrm{m} 2$. The skin and visible mucous membranes are pale. There is dry skin, a positive symptom of «muscle cushion». Peripheral lymph nodes are not enlarged, painless. Swelling of the legs and feet. In the lungs, with percussion, a clear lung sound, auscultatory - vesicular breathing, no wheezing. The boundaries of the heart are within normal limits. Heart sounds are rhythmic, clear. Pulse 76 beats $/ \mathrm{min}$, BP 90/60 mm. Hg Tongue moist, clean with smoothed papillae. The abdomen is swollen, on palpation it is soft, doughy consistency. Ascites is determined. There are no symptoms of peritoneal irritation. The liver protrudes from under the costal arch by $2 \mathrm{~cm}$. The spleen is not palpable. The kidneys are not palpable. The tapping symptom is negative on both sides.

On the side of 12 pairs of cranial nerves without features. In blood tests: iron deficiency anemia $(\mathrm{Hb}-11.7 \mathrm{~g} / \mathrm{l}$, serum iron 4.8 $\mathrm{mmol} / \mathrm{l}$ ), hypoproteinemia (total protein $41.4 \mathrm{~g} / \mathrm{l}$ ), hypoalbuminemia $21.3 \mathrm{~g} / 1$, hypocalcemia $1.74 \mathrm{mmol} / 1$, hypomagnesemia $-0.41 \mathrm{mmol} / 1$, hypertransaminasemia ALT283.2, AST 240.2.Coagulogram: APTT $41.1 \mathrm{sec}$ (norm 25-36.9), INR 2.41 (norm 0.90-1.2), prothrombin time $-26.6 \mathrm{sec}$. Urine and stool analyzes without abnormalities. Immunological study: antibodies to gliadin IgA-120 U/ml, (the norm is up to $12 \mathrm{U} / \mathrm{ml}), \operatorname{IgG} 100 \mathrm{U} / \mathrm{ml}$. Ab to tissue transglutaminase IgA$200, \mathrm{IgG}-144.0 \mathrm{U} / \mathrm{ml}$ (the norm is up to $10 \mathrm{U} / \mathrm{ml}$ ).

Ultrasound of the abdominal organs: Ascites. Enlargement and diffuse changes in the liver of the liver. Chronic cholecystitis. Biliary sludge.

CT enterography: There is no evidence of inflammatory changes in the walls of the small and large intestine. Chronic cholecystitis. A small amount of free fluid in the abdominal cavity and small pelvis.

EGDS: The mucous membrane of the stomach is pink with foci of hyperemia. In the antrum, single erosion up to $0.3 \mathrm{~cm}$ with unchanged mucosa. The mucous membrane of the duodenum is mosaically striated, the villi are smoothed. Conclusion: Chronic erosive gastritis. Endoscopic signs of celiac disease.

Conclusion on the morphological study of biopsy specimens of the duodenal mucosa: Microscopic description: fragments from the distal mucous membrane of the duodenal ulcer: Intestinal villi are absent (V-0), crypt hyperplasia is noted. The surface epithelium with desquamation sites is flattened, with vacuolization of the cytoplasm, the number of goblet cells is reduced. The number of MELs in the surface epithelium and crypt epithelium is increased (65 per 100 epithelial cells). In the lamina propria pronounced lymphoplasmacytic infiltration with an admixture of eosinophils and histiocytes. Conclusion: Marsh IIIC stage celiac disease.

B In this hospitalization, due to severe electrolyte disturbances and the severity of the condition on admission, it was decided to abstain from fibrocolonoscopy. The last colonoscopy from 19.10.17 - examination at $75 \mathrm{~cm}$ to the hepatic flexure - without organic pathology. Irrigoscopy from 03/02/18 - without pathology.

Bolnoy conducted a study of the activity of intestinal carbohydrases according to the biopsy of the lateral part of the duodenum. As can be seen from Table 1, the patient showed a decrease in the activity of all studied enzymes. Such a significant decrease in intestinal enzymes, which carry out the final stage of hydrolysis and absorption of nutrients, indicates a violation of the functional state of the SOTC.

On the basis of the clinical picture of the disease: persistent diarrhea, frolicking a month after childbirth, the rapid onset of malabsorption syndrome: progressive weight loss, iron deficiency anemia, hypoproteinemia with hypoprotinemic edema, ascites; examination results: high values of immunological tests of antibodies to tissue trpanglutamtnase IgA and IgG, the results of morphological examination of the SOTC, which revealed mucosal atrophy at the Marsh IIIC stage, a decrease in the functional capacity of the small intestine in the form of a low level of SOTC carbohydrases, the patient was diagnosed with Celiac disease, newly diagnosed, latent the form. Syndrome of impaired absorption of the 3rd degree of severity with impaired protein, electrolyte, vitamin metabolism. Hypoproteinemia, hypoproteinemic edema, ascites, hypokalemia, hypocalcemia. Irondeficiency anemia. Disaccharidase deficiency Chronic erosive gastritis not associated with HP, subsiding exacerbation. Liver steatosis.

Treatment was carried out: gluten-free diet, correction of metabolic disorders: infusion of albumin and amino acids solution,: albumin $20 \%-200 \mathrm{ml}$ No. 4, aminosteril hepa, electrolyte solutions, iron. Intravenous infusion of glucocorticoids (prednisolone 60mg No. 4) with the transition to oral administration at a dose of $60 \mathrm{mg}$. On the background of therapy, an improvement in the condition was noted: a decrease in stool, a decrease in edema, a normalization of laboratory parameters: the level of hemoglobin, total protein, albumin, blood electrolytes.

\section{Discharged in satisfactory condition with recommendations}

Compliance with a gluten-free diet is strict and lifelong. Continue taking glucocorticoids according to the scheme with decreasing the dose of prednisolone: $60 \mathrm{mg}$ with a gradual decrease in the dose of $5 \mathrm{mg}$ weekly, proton pump inhibitors - omeprazole $20 \mathrm{mg} \times 2$ times a day - while taking prednisolone. Calcium preparations - calcium D3 - for a long time, iron preparations until hemoglobin and serum iron normalization, enzyme preparations during meals, enteroprotector rebamipide $100 \mathrm{mgx} 3$ times a day for 2 months. Dynamic observation at MKSC - repeated hospitalization after 2 months to assess the effectiveness of therapy. After 2.5 months, the patient was admitted to the intestinal department of the A.S. Loginov for dynamic monitoring.

Complaints on admission: hair loss, increased gas production after consuming dairy products. The general condition is satisfactory. 
Weight $62 \mathrm{~kg}$, skin and visible mucous membranes are pink, clean. Peripheral lymph nodes are not enlarged. From the lungs and heart without deviations from the norm. The tongue is moist, clean. The abdomen is not swollen, on palpation it is soft, painless. Stool decorated 1.2 times a day. The kidneys are not palpable. The tapping symptom is negative on both sides. According to laboratory tests, normalization of clinical and biochemical blood tests: hemoglobin $13.0 \mathrm{~g} / 1$, serum iron $32.2 \mathrm{mmol} / 1$, total protein $62 \mathrm{~g} / 1$, albumin $37.3 \mathrm{~g} / 1$, electrolyte levels, transaminases within normal values ... Remained slightly increased level of antibodies to tissue transglutaminase - IgA$26 \mathrm{U} / \mathrm{ml}$ (at a rate of up to $10 \mathrm{U} / \mathrm{ml}$, against indicators in the previous hospitalization - 200U / $\mathrm{ml}$ ).
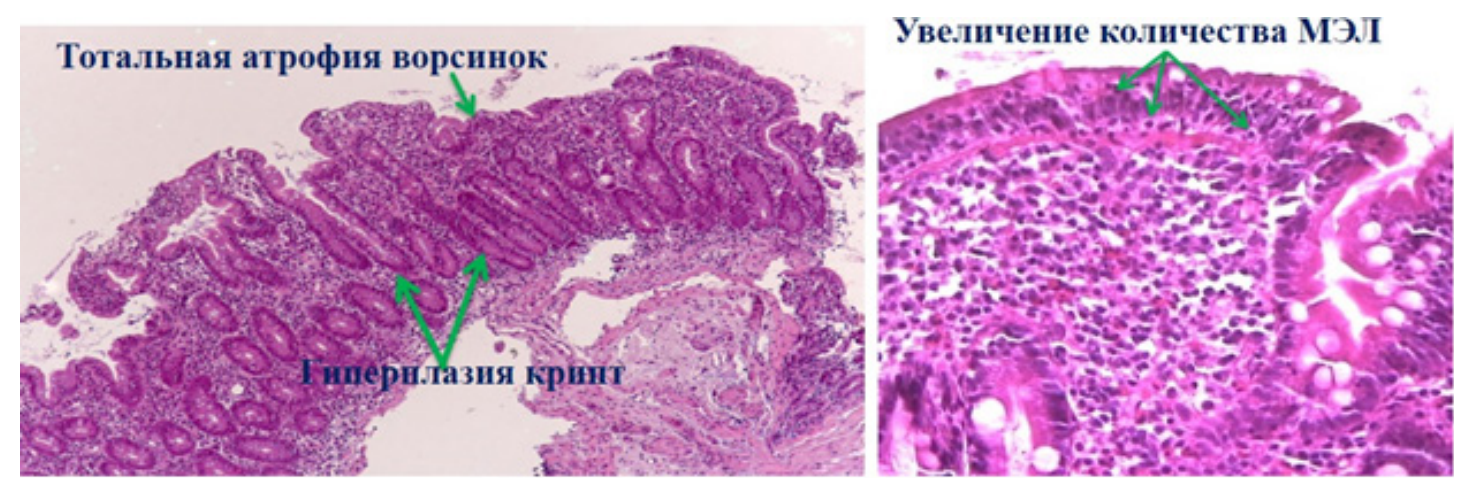

Figure I Morphological picture of SOTK.

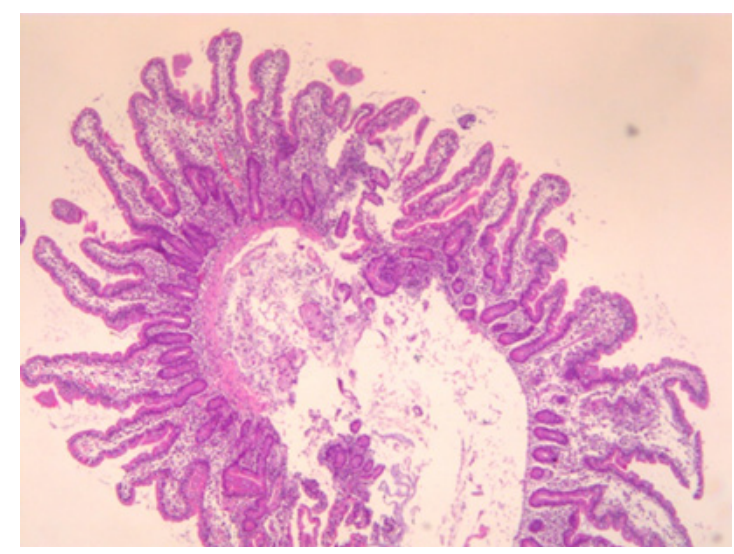

Figure 2 Morphological picture of the mucous membrane during rehospitalization.

Table I Activity of carbohydrases, enzymes of membrane digestion in SOTC

\begin{tabular}{lll}
\hline & $\begin{array}{l}\text { Carbohydrases } \\
\text { level in units }\end{array}$ & Reference values \\
\hline Glucoamylase & 29 & $158-860$ \\
Maltase & 173 & $844-2627$ \\
Sucrose & 12 & $66-206$ \\
Lactase & 3 & $15-67$ \\
\hline
\end{tabular}

According to ultrasound of the abdominal cavity: Diffuse changes in the liver, pancreas. Conducted esophagogastroduodenoscopy, conclusion: Insufficiency of the cardia. Focal gastritis. Duodenitis. A biopsy was taken for morphological examination.

Microscopic description: the specimen contains fragments of the mucous membrane from the distal duodenum. Intestinal villi are high, lined with cells of high columnar epithelium, with the presence of goblet cells. The contours of the brush border are blurred in some areas. The crypts are shallow, in the bottom of the crypts there are a large number of Paneth cells. The villus/crypt ratio is 3:1-4: 1 . The number of MEL (20 per 100 epithelial cells). The lamina propria is unevenly infiltrated by lymphocytes, plasmocytes with a slight admixture of eosinophils. Conclusion: the morphological picture may correspond to gluten enteropathy during treatment with a gluten-free diet. Compared with the primary biopsy, there is a positive trend in the form of an almost complete restoration of the structure of the duodenal mucosa.

Table 2 The level of carbohydrases SOTK after 2 months of adherence to AGD + rebamipide

\begin{tabular}{lll}
\hline & $\begin{array}{l}\text { The level of } \\
\text { carbohydrases in units }\end{array}$ & Reference values \\
\hline Glucoamylase & 820 & $158-860$ \\
Maltase & 1900 & $844-2627$ \\
Sucrose & 272 & $66-206$ \\
Lactase & 13 & $15-67$ \\
\hline
\end{tabular}

As can be seen from the presented table, the activity of the studied carbohydrases increased to normal values, except for lactase, the level of which remained somewhat below normal.

The patient was discharged in a satisfactory condition with a recommendation to strictly follow a gluten-free diet for life. Dynamic observation after 1 year, or in case of deterioration of health.

The peculiarity of this case is that a patient with a severe course of latent celiac disease, occurring with malabsorption syndrome 3 tbsp. severity with hypoportheinemic edema, ascites, severe electrolyte disturbances, anemia, high levels of antibodies to tissue transglutaminase, severe mucosal atrophy corresponding to Marsh IIIC, disaccharidase deficiency, 2 months after the diagnosis and therapy was prescribed, complete remission occurred - clinical, morphological, functional. This may be due to the rapid appointment of adequate therapy, which included (the fact that immediately after admission to the intestinal department of the MSCC the patient was prescribed intensive therapy :) gluten-free diet, glucocorticoids, correction of metabolic disorders. With strict adherence to a gluten- 
free diet, clinical remission, normalization of immunological tests usually occur within 6 to 12 months from the start of treatment, and complete restoration of the morphological structure of the SOTC and its functional ability - after 1.5-3 years from the start of therapy. After the first hospitalization, the patient was recommended the drug rebamipide, which she took for a month. It can be assumed that taking rebamipide was the reason for the rapid onset of clinical remission, complete restoration of the morphological structure of the SOTC and its functional state.

\section{Conclusion}

An example of a patient with latent celiac disease with severe malabsorption syndrome, high values of immunological tests, total atrophy of the mucous membrane of the small intestine (Marsh IIIC stage), severe membrane digestion disorders was demonstrated. The patient, against the background of strict adherence to a gluten-free diet, taking rebamipide, 2 months after the start of therapy, a complete remission occurred: clinical, morphological, normalization of the functional state of the mucous membrane of the small intestine. It can be assumed that such a rapid onset of complete remission occurred in connection with the intake of a cytoprotective agent - rebamipide.

\section{Acknowledgments}

None.

\section{Conflicts of interest}

The authors declare that there are no conflicts of interest.

\section{Funding}

None.

\section{References}

1. Parfenov AI, Maev IV, Baranov AA, et al. All-Russian consensus on the diagnosis and treatment of celiac disease in children and adults. Almanac of Clinical Medicine. Ter Arkh. 2017;89(3):94-107.
2. Parfenov AI. Celiac disease. Evolution of ideas about prevalence, clinical manifestations and significance of etiotropic therapy. $M$ Anaharis. 2007;372.

3. Kroums LM, Sabelnikova EA. Chronic diseases of the small intestine (clinical picture, diagnosis, treatment). Moscow. 2014;95.

4. Parfenov AI, Krums LM. Malabsorption, the leading clinical sign of diseases of the small intestine. Ter Arkh. 2016;88(8):7-9.

5. Akhmadullina OV, Sabelnikova EA, Belostotsky NI, et al. Enzymatic activity of the mucous membrane of the small intestine in celiac disease patients following a gluten-free diet. Doctor Ru. 2017;2(131):22-25.

6. Stimanenkov VI, Tikhonov SV. Rebamipide - new possibilities of gastroenterology. Ter Arkh. 2015;87(12s):134-137.

7. 7.Yakovenko EP, Agafonova NA, Ivanova AN, et al. The cytoprotector rebamipide in the treatment of inflammatory and erosive-ulcerative lesions of the gastrointestinal tract. Ter Arkh. 2016;88(4):88-92.

8. Mizukami K, Murakami K, FbeT. etall. Ashirin induced small bowel injuries and the preventive effect of rebamipid. Word J Gastroenterol. 2011;17(46):5117-5122.

9. Zhang S, Qing Q, Bai Y. Pebamipide helps defend against nonsteroidal antiinflamatory drags indced gastroeneropathy. A systematic review and meta-analysis. Dig Dis Sci. 2013;58(7):1991-2000.

10. Tarnawsri AS, Chai J, Pai R, et al. Rebamipid activates gene encoding angiogenic growth factorsand $\mathrm{COX} 2$ and stimulates angiogenesis: a key to its ulcer healing action?" Dig Dis Sci. 2004;49(2):202-209.

11. Parfenov AI, Belostotsky NI, Khomeriki SG, et al. Rebamipide increases the activity of disaccharidases in patients with enteropathy with impaired membrane digestion. Pilot study. Ter Arkh. 2019;91(2):25-31. 